\title{
Design, chemical synthesis and evaluation of antimicrobial activity peptide conjugates of lactoferricin analogues and antibiotics
}

\author{
Natalia Ptaszyńska ${ }^{1}$, Katarzyna Olkiewicz ${ }^{1}$, Joanna Okońska ${ }^{1}$, Anna Łęgowska ${ }^{1}$, Katarzyna Gucwa ${ }^{2}$, Agata \\ Gitlin-Domagalska $^{1}$, Dawid Dębowski ${ }^{1}$, Sławomir Milewski ${ }^{2}$, Krzysztof Rolka $^{1}$ \\ ${ }^{1}$ Univesity of Gdańsk, Faculty of Chemistry, Departmentof Molecular Biochemistry, Poland \\ ${ }^{2}$ Gdańsk University of Technology, Faculty of Chemistry, Departmentof Drug Technology and Biochemistry, Poland
}

https://doi.org/10.17952/35EPS.2018.306

\section{Introduction}

Despite the increasing need for antibiotics to fight infectious diseases, fewer new antibiotics are available on the market. Modification or combination of existing antibiotics to improve their efficacy is one of a promising strategy. In this broad field, peptide - drug conjugates linked by non-cleavable or intracellular cleavable structures have evolved as highly promising agents.

Here we report synthesis and biological investigations of a series of peptide conjugates composed of modified bovine lactoferricin (LFcinB) truncated analogues and three antibiotics, ciprofloxacin (CIP), levofloxacin (LVX) and fluconazole (FLC) commonly used in medical practice. The first two belong to the class of fluoroquinolone displaying broad spectrum of antimicrobial activity against both Gram-positive and Gram-negative bacteria. They inhibit DNA gyrase and topoisomerases. FLC, similarly to other azole drugs, inhibits the lanosterol $14 \alpha$-demethylase and decreases the level of ergosterol required for the integrity of cell membrane [1]. LFcinB is an antimicrobial peptide isolated in 1992 by Bellamy et al. [2] from bovine milk, representing 17-41 fragment of bovine, multifunctional iron-binding glycoprotein, lactoferrin (LF). LFcinB displays a broad spectrum of antibacterial and antifungal activity and also cooperative effect with azole types of antifungal agents [3]. Our intention was to design a new class of antimicrobial compounds. Chemical structures of constituent compounds and peptide conjugates are shown in Fig. 1.

\section{Results and Discussion}

A series of eight new peptide conjugates containing three above mentioned antibiotics was obtained. Four different approaches were applied to couple a peptide and the antimicrobial agent. We developed an optimized conditions for coupling of LVX via its carboxylic group, CIP via its carboxylic or amino group to peptide chain using solid phase peptide synthesis. In the last step of synthesis, both drugs were attached through their carboxyl groups to the N-terminal amino group of peptidyl-resin, whereby LVX-LFcinB(2-11)-NH 2 (I) and CIP-LFcinB(2-11)- $\mathrm{NH}_{2}$ (II) were obtained. CIP was also coupled to the peptide by the method utilized for the synthesis of peptoids, named submonomeric [4]. In this case, the covalent bond was formed in reaction of the secondary amino group of CIP and bromoacetylated peptidyl-resin, yielding CIP-CH2-COLFcinB(2-11)- $\mathrm{NH}_{2}$ (III). In the next two conjugate, CIP-Cys-S-S-LFcinB(2-11)- $\mathrm{NH}_{2}$ (IV) and CIP-Cys-S-SNle-LFcinB- $\mathrm{NH}_{2}(\mathrm{~V})$ intermolecular disulfide bridge was used as a linker between peptide and CIP, formed by the thiol group of peptide Cys present in position 3 and thiol group of Cys attached to CIP amino group. In both conjugates (IV and V) a disulfide bridge was formed in solution phase with the help of S-(3-nitro-2pyridylsulfanyl) (Npys) group introduced on Cys attached to CIP. In the case of fluconazole-based conjugates VI - VIII contained LFcinB(2-11)- $\mathrm{NH}_{2}$, Nle-LFcinB and Nle-LFcinB-NH $\mathrm{N}_{2}$, respectively, a "click chemistry" method was used to attach this antifungal agent derivative to a peptidic component. FLC-based conjugates attached through their N-termini to the nitrogen atom of FLC and methylene-carbonyl moiety was used as a linker. This required synthesis of the FLC precursor which was obtained by the method described by Pore et al. [5]. 


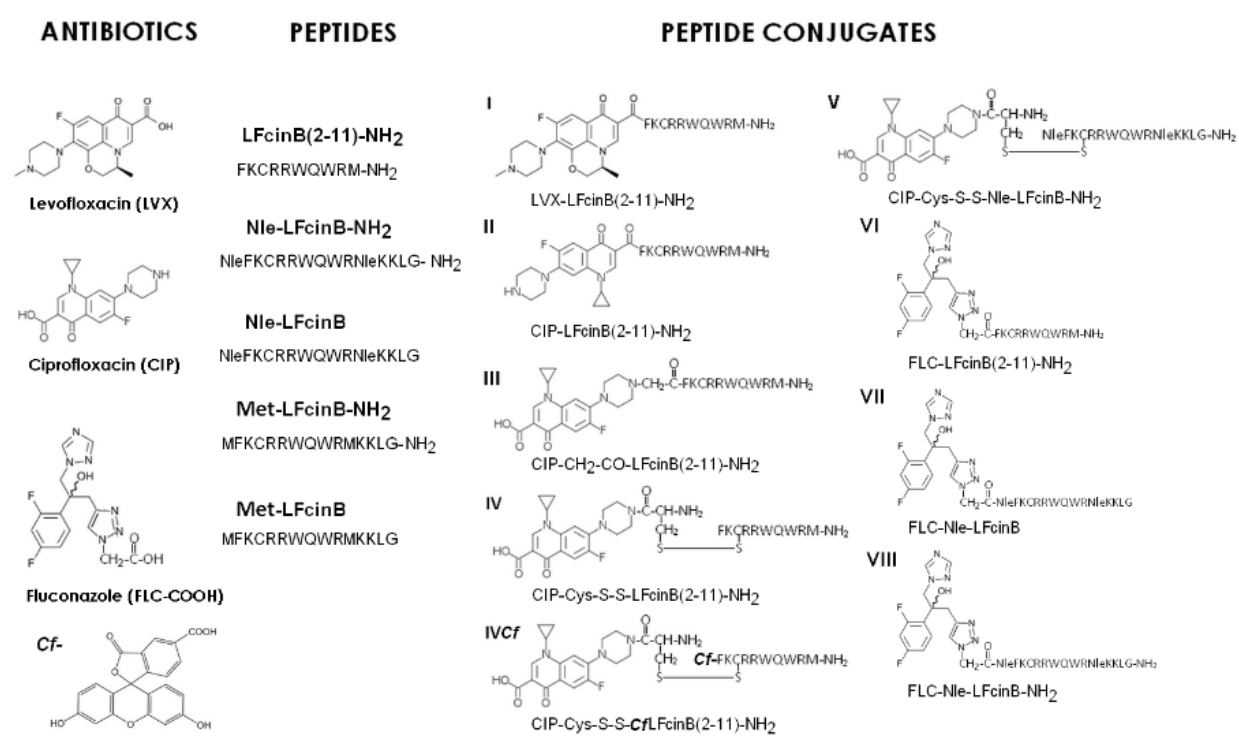

Figure 1: Chemical structures of peptide conjugates, constituent peptides and antibiotics.

All synthesised conjugates and their constituent compounds were investigated for fungicidal activity against five fungal stains C. albicans ATCC 10231, C. krusei DSM 6128, C. parapsilosis DSM 5784, C. glabrata DSM 11226 and $C$. tropicalis CZD 519 and for antibacterial activity against six bacterial strains, four Grampositive ( S. aureus ATCC 25923, S. epidermidis ATCC 12228, B. subtilis ATCC 6633, B. cereus PCM 2003), two Gram-negative (E.coli ATCC 25922, P. aeruginosaATCC 27857). Constituent peptides displayed weak inhibitory activity against tested strains. The synthesized conjugates, except the conjugate (VII), displayed stronger antifungal activity than the constituent peptides, and in the most cases, than CIP and LVX. The most active appeared to be conjugate (I) composed of LVX and the shortest peptide LFcinB(2-11)- $\mathrm{NH}_{2}$ which inhibited the growth of $C$. krusei and C. parapsilosis. The determined values of $\mathrm{MIC}_{50}$ were 12.5 and $1.56 \mu \mathrm{g} / \mathrm{mL}$, respectively. All constituent peptides exhibited antibacterial activity, especially against Gram-positive bacteria. The lowest $\mathrm{MIC}_{50}$ and MIC90 values were determined for $\mathrm{LFc}$ inB(2-11)- $\mathrm{NH}_{2}$ $(3.13,6.25 \mu \mathrm{g} / \mathrm{ml})$ and Nle-LFcinB- $\mathrm{NH}_{2}(3.13 \mu \mathrm{g} / \mathrm{ml})$ against $\mathrm{S}$. epidermidis. These two peptides were also active against $S$. aureus and $E$. coli . Fluorescently labeled CIP-Cys-S-S-Cf-LFcinB(2-11)-NH 2 (IVCf) as well as its constituent Cf- $\mathrm{LF} \operatorname{cinB}(2-11)-\mathrm{NH}_{2}$ are able to penetrate both $C$. albicans and $\mathrm{S}$. epidermidis. However, despite rapid and effective internalization they do not show antifungal activity.

Cytotoxicity of conjugates, constituent peptide and drugs (LVX, CIP, FLC) was assessed against four human cell lines: non-cancer cell line of embryonic kidney (HEK 293) and three cancer cell lines: nonsmall cell lung cancer (A549), breast cancer (BT-20) and acute myeloblastic leukemia (HL-60). Cytotoxicity assays on cancer and non-cancer cell lines showed that the proposed conjugates induce a relatively low cytotoxic effect in reference to antibiotics widely used in clinic (except FCL which was least toxic). Noncancer cell line was the most resistant to the action of the tested compounds, where cell growth was virtually not affected up to the concentration of $250 \mu \mathrm{M}$.

ROS generation by conjugates and their constituents was tested on non-cancer cell-line HEK 293 and cancer cell line HL-60. In neither of tested conjugates the amount of ROS generated was higher than $25 \%$. In contrast CIP and LVX caused production of ROS in more than $50 \%$ of the cells. The differences were more pronounced in case of cancer cells where the observed amount of ROS generated under influence of conjugates was comparable to the negative control (not treated with any compound) or slightly elevated, while in the case of antibiotics the amount of ROS was on the level of positive control (H2O2) or even higher.

Within this study, eight conjugates of antimicrobials, CIP, LVX and FLC and the LFcinB peptide were synthesized and evaluated for their antimicrobial activity. Three FLC-based conjugates, to our knowledge, are the first examples of peptide conjugates of this antifungal agent reported in literature. Summarizing the results presenting above we would like to stress that some of the obtained conjugates showed promising antibacterial activity, especially against Gram-positive bacteria, within which S. epidermidis was the most sensitive. The most active 
ones were conjugates containing CIP attached to the peptide by the redox-sensitive disulfide bridge. They displayed not only higher efficacy than constituent peptides, but also broader spectrum of activity, inhibiting the growth of other strains. The obtained results indicate an important role of a linker, especially a disulfide bridge, used to couple drug and peptide. In all three conjugates (III-V), CIP was attached through its secondary amino group, but the highest antimicrobial activity showed those with disulfide bridge. The primary novelty of this work is the activity of CIP and LVX based conjugates against Candida yeast. Studies of CIP/LVX with peptide combinations against Candida yeast did not indicate the possibility of synergistic effects which also indicate an important role of a linker, especially a disulfide bridge, used to couple drug and peptide. Taking all above into consideration, also low or moderate side effects (toxicity and ROS generation) of studied conjugates, we find that proposed conjugates are promising led structures for development of new antibacterial drugs.

\section{Acknowledgements}

This work was supported by theNational Science Centre (NCN) under grant No UMO-2016/21/B/ST5/00101.

\section{References}

[1] Kabir M.A., Ahmad Z., ISRN Preventive Medicine, https://doi.org/10.5402/2013/763628 (2013).

[2] Bellamy W., Takase M., Yamauchi K., Wakabayashi H., Kawase K., Tomita M. Biochim. Biophys. Acta 1121, 130-136 (1992)

[3] Wakabayashi H., Abe S., Teraguchi S., Hayasawa H., Yamaguchi H. Antimicrob Agents Chemother, 42, 1587-1591 (1998), DOI: 10.1128/AAC.42.7.1587.

[4] Ł ęgowska A., Dębowski D., Łukajtis R., Wysocka M., Czaplewski C., Lesner A., Rolka K. Bioorg. Med. Chem. 18, 8188-8193 (2010)

[5] Pore V. S., Aher N. G., Kumar M., Shukla P. K. Tetrahedron62, 11178-11186 (2006) 